\title{
Study of propagation of linear and non-linear Alfvén-gravity waves in rotating medium
}

\author{
L.N. Achala ${ }^{\mathrm{a}, *}$, M. Venkatachalappa ${ }^{\mathrm{b}}$ \\ ${ }^{a}$ P. G. Department of Mathematics and Research Centre in Applied Mathematics, MES College, Bangalore 560003, India \\ ${ }^{\mathrm{b}}$ Department of Mathematics, Central College, Bangalore University, Bangalore 560 001, India
}

\section{A R T I C L E I N F O}

\section{Article history:}

Received 21 September 2009

Received in revised form

12 April 2010

Accepted 5 April 2011

Available online 21 April 2011

Keywords:

Travelling waves

Stratified flows

Internal gravity waves

Conducting fluid

Phase-space

Rotating flows

\begin{abstract}
A B S T R A C T
Travelling waves in an incompressible, infinitely conducting, inviscid fluid of variable density are investigated under the influence of a horizontal magnetic field and Coriolis force. Periodic solutions are found in the limit of infinite vertical wave length. Phase diagrams are drawn to show the solution.
\end{abstract}

(c) 2011 Published by Elsevier Ltd.

\section{Introduction}

It has been established that disturbances in uniformly rotating incompressible liquids can propagate as wave motions. Many aspects of upper atmospheric dynamics and ionospheric irregularities may be explained in terms of internal gravity waves which are responsible for transfer of momentum and energy from one region to another. The effect of Coriolis force due to Earth's rotation plays an important role in the understanding this mechanism. Venkatachalappa et al. [1] have studied the propagation of linear and non-linear travelling waves in a compressible rotating atmosphere. Venkatachalappa et al. [2] investigated the propagation of linear and non-linear travelling waves in an exponentially stratified incompressible rotating fluids. Venkatachalappa et al. [3] have studied the propagation of linear and non-linear hydromagnetic waves in an exponentially stratified non-rotating incompressible medium. In the present paper we analyse the effect of rotation and magnetic field on linear and non-linear internal gravity waves propagating in an exponentially stratified incompressible and infinitely conducting fluid. The waves under study are governed by a system of nine non-linear inhomogeneous PDEs. We seek travelling wave solutions of this system. The resulting ODE system is reduced via some first integrals to one of third order and analysed in phase-space.

\footnotetext{
* Corresponding author.

E-mail address: anargund@hotmail.com (L.N. Achala).
}

The scheme of the present paper is as follows. Section 2 contains basic equations and travelling wave formulation. Phaseplane analysis for both linear and non-linear waves is given in Section 3 and the conclusion of the present study in Section 4.

\section{Basic quations}

We consider Cartesian coordinate system with $x$ and $y$ axes in the horizontal plane and the $z$-axis along the vertical direction. We study quasi-simple waves in an incompressible, infinitely conducting stratified fluid rotating with a uniform angular velocity $\Omega$ about vertical axis in the presence of an applied horizontal magnetic field. The equations governing the unsteady system are

$\rho\left[\frac{D \vec{q}}{D t}+2 \vec{\Omega} X \vec{q}\right]+\nabla P-\rho \vec{g}-\mu(\vec{H} \cdot \nabla) \vec{H}=0$,

$\frac{D \rho}{D t}=0$,

$\nabla \cdot \vec{q}=0$

$\frac{D \vec{H}}{D t}-(\vec{H} \cdot \nabla) \vec{q}=0$,

$\nabla \cdot \vec{H}=0$, 
where $\frac{D}{D t}=\frac{\partial}{\partial t}+u \frac{\partial}{\partial x}+v \frac{\partial}{\partial y}+w \frac{\partial}{\partial z}, P=p+\mu H^{2} / 2$ is the total pressure, $\vec{q}$ with components $(u, v, w)$ is the fluid velocity, $p$ is the hydrodynamic pressure, $\rho$ is the density, $\vec{g}$ is the acceleration due to gravity, $\vec{\Omega}$ is the angular velocity, $\vec{H}$ is the magnetic field with components $H_{x}, H_{y}, H_{z}$ and $\mu$ is the magnetic permiability. We assume that the undisturbed fluid has density $\rho_{0}(z)$ and an applied horizontal magnetic field $H_{0}(z)$ given by

$\rho_{0}(z)=\rho_{c} \exp (-z / H), \quad H_{0}(z)=H_{c} \exp (-z / 2 H)$,

where $H$ is the scale height and $\rho_{c}$ and $H_{c}$ are the reference density and reference magnetic field at $z=0$, respectively. From (1) we find that

$p_{0}(z)=p_{c} \exp (-z / H) \quad$ where $p_{c}=g H \rho_{c}$.

\section{Travelling wave solution}

We non-dimensionalise Eqs. (1)-(5) using $H,(g / H)^{12},(g H)^{12}$, $p_{c} e^{(-z / H)}, \rho_{c} e^{(-z / H)}$ and $H_{c} e^{(-z / 2 H)}$ as the scales for length, time, velocity, pressure, density and magnetic field, respectively. We seek travelling wave solutions in the form $u, v, w, \rho, P, H_{x}, H_{y}, H_{z}$ are functions of $\varphi$ where

$\varphi=\frac{x}{\lambda_{1}}+\frac{y}{\lambda_{2}}+\frac{z}{\lambda_{3}}-t$,

and $\lambda_{1}, \lambda_{2}$ and $\lambda_{3}$ are wave lengths in $x, y$ and $z$ directions, respectively. The initial conditions are

$u=v=w=H_{y}=H_{z}=0, \quad p=\rho=H_{x}=1$.

Resulting equations are

$E u_{\varphi}-2 \Omega v+\frac{1}{\rho \lambda_{1}} P_{\varphi}-\frac{A^{2}}{\rho}\left(B\left(H_{\chi}\right)_{\varphi}-\frac{H_{x} H_{z}}{2}\right)=0$,

$E v_{\varphi}+2 \Omega u+\frac{1}{\rho \lambda_{2}} P_{\varphi}-\frac{A^{2}}{\rho}\left(B\left(H_{y}\right)_{\varphi}-\frac{H_{y} H_{z}}{2}\right)=0$,

$E w_{\varphi}+\frac{1}{\rho \lambda_{3}} P_{\varphi}-\frac{P}{\rho}+1-\frac{A^{2}}{\rho}\left(B\left(H_{z}\right)_{\varphi}-\frac{H_{z}^{2}}{2}\right)=0$,

$E \rho_{\varphi}-\rho w=0$

$\frac{u_{\varphi}}{\lambda_{1}}+\frac{v_{\varphi}}{\lambda_{2}}+\frac{w_{\varphi}}{\lambda_{3}}=0$

$E\left(H_{x}\right)_{\varphi}-B u_{\varphi}-\frac{w H_{x}}{2}=0$,

$E\left(H_{y}\right)_{\varphi}-B v_{\varphi}-\frac{w H_{y}}{2}=0$,

$E\left(H_{z}\right)_{\varphi}-B w_{\varphi}-\frac{w H_{z}}{2}=0$,

$\frac{\left(H_{x}\right)_{\varphi}}{\lambda_{1}}+\frac{\left(H_{y}\right)_{\varphi}}{\lambda_{2}}+\frac{\left(H_{z}\right)_{\varphi}}{\lambda_{3}}-\frac{H_{z}}{2}=0$,

where $E=\frac{u}{\lambda_{1}}+\frac{v}{\lambda_{2}}+\frac{w}{\lambda_{3}}-1$ and $A^{2}=\frac{\mu H_{c}^{2}}{\rho_{c} g H}$ represents non-dimensional Alfven velocity and $B=\frac{H_{x}}{\lambda_{1}}+\frac{H_{y}}{\lambda_{2}}+\frac{H_{z}}{\lambda_{3}}$, respectively. Integrating (14) using the initial conditions (9) and suitably combining Eqs. (15)-(18), we get the following three first integrals:

$\frac{u}{\lambda_{1}}+\frac{v}{\lambda_{2}}+\frac{w}{\lambda_{3}}=0$,
$\frac{H_{x}}{\lambda_{1}}+\frac{H_{y}}{\lambda_{2}}+\frac{H_{z}}{\lambda_{3}}=-\frac{H_{z}}{w}$,

$\frac{H_{z}^{2}}{\rho w^{2}}=Q \quad$ (a constant)

Writing $T=\frac{u}{\lambda_{2}}-\frac{v}{\lambda_{1}}$ and using the first integrals (19)-(21) in (10)-(18) we get a third order system:

$w_{\varphi}=\frac{-\bar{n}(K-1)-2 \Omega T}{n \lambda_{3}\left(1-A^{2} Q\right)}, \quad K_{\varphi}=K w-\frac{(1-K)}{n \lambda_{3}}-\frac{2 \Omega T}{n}$,

$T_{\varphi}=\frac{2 \Omega w}{\lambda_{3}\left(1-A^{2} Q\right)}$

where

$K=\frac{P}{\rho}, \quad n=\frac{1}{\lambda_{1}^{2}}+\frac{1}{\lambda_{2}^{2}}+\frac{1}{\lambda_{3}^{2}}, \quad \bar{n}=\frac{1}{\lambda_{1}^{2}}+\frac{1}{\lambda_{2}^{2}}$.

The only singular point of system $(22)$ in $(T, w, K)$ space is

$T=0, \quad K=1, \quad w=0$.

It does not seem possible to solve system (22) in a closed form. We first analyse the solution for some limiting cases.

\subsection{Linear system}

We linearise system (22) about the singular point (24). The linearised form of $(22)$ is

$T_{\varphi}^{\prime}=\frac{2 \Omega w^{\prime}}{\lambda_{3}\left(1-A^{2} Q\right)}, \quad w_{\varphi}^{\prime}=\frac{-1}{n\left(1-A^{2} Q\right)}\left(\frac{2 \Omega T^{\prime}}{\lambda_{3}}+\bar{n} K^{\prime}\right)$,

$K_{\varphi}^{\prime}=w^{\prime}+\frac{K^{\prime}}{n \lambda_{3}}-\frac{2 \Omega T^{\prime}}{n}$,

where primed quantities are perturbations over the equilibrium conditions (24), assumed to be small. The characteristic equation of $(25)$ is

$\lambda^{3}+a_{2} \lambda^{2}+a_{1} \lambda+a_{0}=0$,

where

$a_{0}=-\frac{4 \Omega^{2}}{n \lambda_{3}\left(1-A^{2} Q\right)^{2}}, \quad a_{1}=\frac{4 \Omega^{2}}{n \lambda_{3}^{2}\left(1-A^{2} Q\right)^{2}}+\frac{\bar{n}}{n\left(1-A^{2} Q\right)}$,

$a_{2}=-\frac{1}{n \lambda_{3}}$.

Eq. (26) has a pair of pure imaginary roots, $i \sqrt{a_{1}}$ and $-i \sqrt{a_{1}}$, and a real root $-a_{2}$ if

$4 \Omega^{2}=\left(1-A^{2} Q\right), \quad 4 \Omega^{2}>\bar{n} \lambda_{3}^{2}\left(A^{2} Q-1\right)$

Condition (28) is satisfied if $A^{2} Q<1$ and either $\Omega^{2}=\frac{\left(1-A^{2} Q\right)}{4}$ or $\lambda_{3} \rightarrow \infty$ or $n \rightarrow \infty$, Thus, propagating linear waves, in general do not exist in a rotating stratified incompressible, infinitely conducting fluid. The phase curves in $\left(w^{\prime}, k^{\prime}\right)$ are depicted in

(i) $\Omega \rightarrow 0$ :

When we consider a non-rotating system, that is, when $\Omega \rightarrow$ 0 , system (25) reduce to

$T^{\prime}=0, w_{\varphi}^{\prime}=\frac{-\bar{n} K^{\prime}}{n\left(1-A^{2} Q\right)}, \quad K_{\varphi}^{\prime}=w^{\prime}+\frac{K^{\prime}}{n \lambda_{3}}$.

Eq. (29) is the same as those obtained by Venkatachalappa et al. [1] in the study of hydromagnetic waves in an exponentially stratified incompressible fluid. In the nonconducting limit, that is, as $A^{2} Q \rightarrow 0$, Eq. (29) is identical to those obtained by Venkatachalappa et al. [2] for the incompressible rotating fluids in non-rotating limit. The 
characteristic equation for (29) is (Fig. 1)

$n \lambda_{3}\left(1-A^{2} Q\right) \lambda^{2}+\left(1-A^{2} Q\right) \lambda+\bar{n} \lambda_{3}=0$.

The roots of (30) are real if either $A^{2} Q>1$ or $A^{2} Q<1-4 n \bar{n} \lambda_{3}^{2}$, equal if $A^{2} Q=1$, complex if $1-4 \bar{n} n \lambda_{3}^{2}<A^{2} Q<1$, and pure imaginary when either $\lambda_{3} \rightarrow \infty$ and $A^{2} Q<1$. These limiting cases are shown in Figs. 2 and 3 for both $\lambda_{3}$ finite and infinite (conducting).

(ii) $\lambda_{3} \rightarrow \infty, \Omega \neq 0$ :

This case corresponds to horizontally propagating linear waves (see also (i) above). Using initial condition (24), system (25) reduces to

$T^{\prime}=0, \quad w_{\varphi}^{\prime}=\frac{-K^{\prime}}{\left(1-A^{2} Q\right)}, \quad K_{\varphi}^{\prime}=w^{\prime}$.

Eq. (31) are identicle to those obtained by Venkatachalappa et al. [3] for incompressible infinitely conducting fluid. When we consider the non-conducting limit, that is, in the limit $A^{2} Q \rightarrow 0$, Eq. (31) is identicle to those obtained by Venkatachalappa et al. [1] if we let $\gamma \rightarrow \infty$ in their equations and are the same as those obtained by Venkatachalappa et al. [2] for incompressible rotating fluid if we let $\Omega \rightarrow 0$ in their equations. The phase curves of (31) are given by

$\frac{w^{\prime 2}}{2}+\frac{K^{\prime 2}}{2\left(1-A^{2} Q\right)}=c$

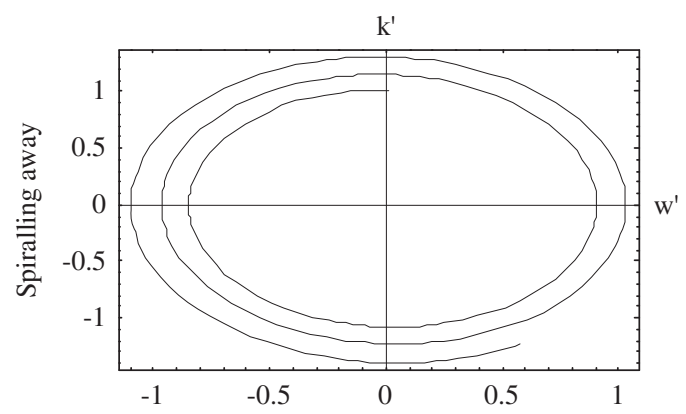

Fig. 1. $\Omega=0.01, A^{2} Q=0.01, \lambda_{1}=\lambda_{2}=\lambda_{3}=0.1$

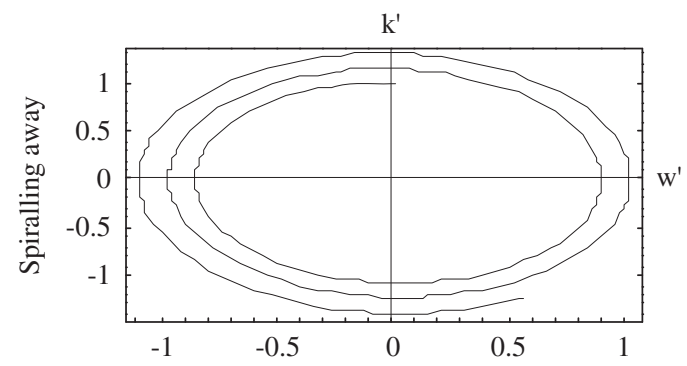

Fig. 2. $\Omega=0.0, A^{2} Q=0.01, \lambda_{1}=\lambda_{2}=0.1, \lambda_{3}=0.1$.

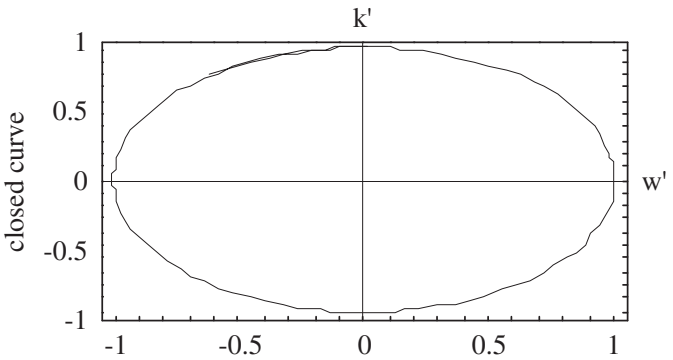

Fig. 3. $\Omega=0.0, A^{2} Q=0.01, \lambda_{1}=\lambda_{2}=0.1, \lambda_{3} \rightarrow \infty$. where $c$ is the constant of integration. Eq. (32) represents an ellipse if $A^{2} Q<1$, and a hyperbola if $A^{2} Q>1$ provided $c>0$. Thus linear waves are periodic when they propagate horizontally, the periodic nature is independent of rotation and wave numbers in the other two directions. The results corresponding to $\lambda_{3} \rightarrow \infty$ are shown in Fig. 4 in $\left(w^{\prime}, k^{\prime}\right)$ plane and Figs. 5 and 6 represent the variation of $K^{\prime}$ and $w^{\prime}$ with $\varphi$. We observe that the periodic nature of the solution is independent of wave numbers. This is in contrast to the case for the compressible rotating system. For the latter, Venkatachalappa et al. [1] have observed that the conditions for the existence of horizontally propagating periodic waves are

$\bar{n}<n_{1}, \quad \bar{n}>n_{2}$,

where $n_{1}=\min \left[\frac{1}{\gamma}, \frac{4 \Omega^{2}}{(\gamma-1)}\right], n_{2}=\max \left[\frac{1}{\gamma}, \frac{4 \Omega^{2}}{(\gamma-1)}\right], \bar{n}=\frac{1}{\lambda_{1}^{2}}+\frac{1}{\lambda_{2}^{2}}$. Thus periodic waves are possible only for the range of wave numbers given by (33). Thus there exists a window of non-periodicity in the range of $n_{1}<\bar{n}<n_{2}$. However, in the incompressible limit $\gamma \rightarrow \infty, n_{1}$ and $n_{2}$ tend to zero; therefore, the window of non-periodicity disappears and we always have periodic solutions in the linear limit as $\lambda_{3} \rightarrow \infty$. It is clear from (28) that, in the limit $\lambda_{3} \rightarrow \infty$, the conditions for periodicity become independent of rotation provided that $A^{2} Q<1$. However, this picture changes as non-linear effects are taken into account (see Section 3.2).

(iii) $\lambda_{1} \rightarrow 0$ or $\lambda_{2} \rightarrow 0$ :

When we consider either of the limits $\lambda_{1} \rightarrow 0, \lambda_{2} \rightarrow 0$, Eq. (25) becomes

$T_{\varphi}^{\prime}=\frac{2 \Omega w^{\prime}}{\lambda_{3}\left(1-A^{2} Q\right)}, \quad w_{\varphi}^{\prime}=\frac{-K^{\prime}}{\left(1-A^{2} Q\right)}, \quad K_{\varphi}^{\prime}=w^{\prime}$.

From (34), we get the integral

$T^{\prime}=\frac{2 \Omega\left(K^{\prime}-1\right)}{\lambda_{3}\left(1-A^{2} Q\right)}$,

if we use the initial conditions (24). The characteristic Eq. (30) now becomes

$\lambda\left(\lambda^{2}+1\right)=0$.

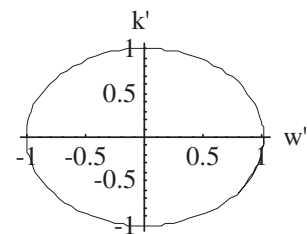

Fig. 4. $\Omega=0.01, A^{2} Q=0.01, \lambda_{1}=\lambda_{2}=0.1, \lambda_{3} \rightarrow \infty$ (periodic solution)

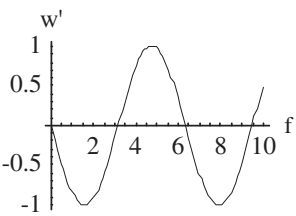

Fig. 5. $\Omega=0.01, A^{2} Q=0.01, \lambda_{1}=\lambda_{2}=0.1, \lambda_{3} \rightarrow \infty$ (periodic solution)

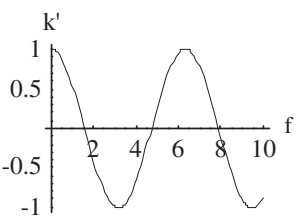

Fig. 6. $\Omega=0.01, A^{2} Q=0.01, \lambda_{1}=\lambda_{2}=0.1, \lambda_{3} \rightarrow \infty$ (periodic solution). 
The three roots of (36) are 0 and $\pm i$. The zero root corresponds to aperiodic solution and the purely imaginary roots give periodic solutions. The nature and behaviour of the solution curves in $\left(w^{\prime}, K^{\prime}\right)$ are similar to that for the case $\lambda_{3} \rightarrow \infty$. Eq. (35) gives linear relation between $T^{\prime}$ and $K^{\prime}$.

\section{2. $\quad$ Non-linear travelling waves}

The non-linear self propagating waves are governed by system (12). It does not seem possible to find an explicit solution for this system. We solve them numerically; the results are shown in Fig. 7 and 8 in which space curve shows periodic solution for $\lambda_{3} \rightarrow$ $\infty$. We observe that the phase curves are in general not closed. We study this system for the following limiting cases.

(i) $\Omega \rightarrow 0$ :

For non-rotating flows, that is, when $\Omega \rightarrow 0$, system (22) reduce to

$w_{\varphi}=\frac{-\bar{n}(K-1)}{n\left(1-A^{2} Q\right)}, \quad K_{\varphi}=w K-\frac{(1-K)}{n \lambda_{3}}$.

The only singular point of system (37) is again given by (24). Eq. (37) are solved numerically and the phase curves in the limit $A^{2} Q \rightarrow 0$ coincides with that obtained by Venkatachalappa et al. [2] for the incompressible non-conducting case and Seshadri and Sachdev [4] if we take the limit $\gamma \rightarrow \infty$ in the latter. Seshadri and Sachdev showed that their system of equations admit only parallel plane wave solutions. They also obtained periodic solutions when there is no propagation in the vertical direction. Seshadri and Sachdev [4] obtained three singular points which coalesce with (24) in the incompressible limit $\gamma \rightarrow \infty$. Thus we observe that

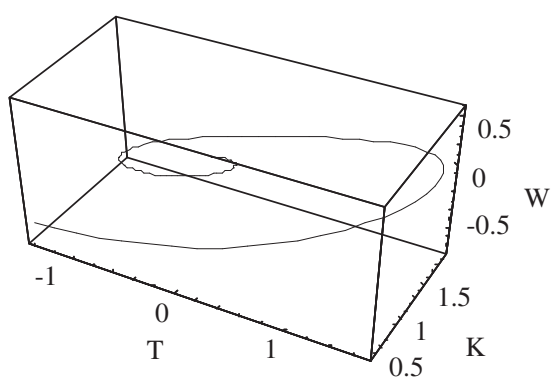

Fig.7. $\Omega=0.01, A^{2} Q=0.01, \lambda_{1}=\lambda_{2}=\lambda_{3}=0.1$ (spiralling away).

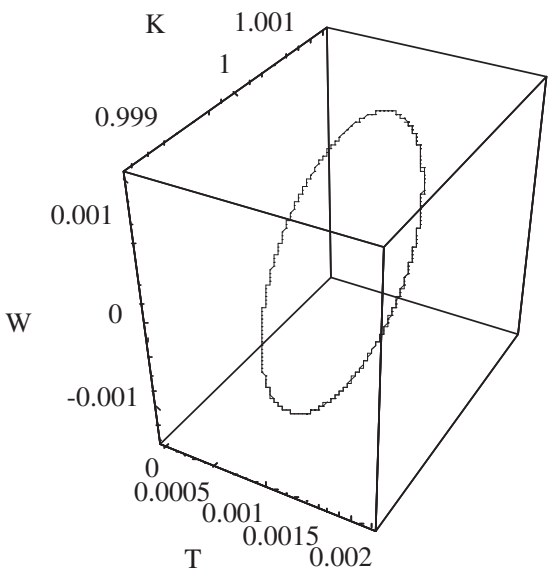

Fig.8. $\Omega=0.01, A^{2} Q=0.01, \lambda_{1}=\lambda_{2}=0.1, \lambda_{3} \rightarrow \infty$ closed curve. rotation and magnetic field effects do not give rise to any new equilibrium states.

(ii) $\lambda_{3} \rightarrow \infty, \Omega \neq 0$ :

When we consider only horizontally propagating waves, that is, the limiting case $\lambda_{3} \rightarrow \infty$, Eq. (22) reduce to

$T=0, w_{\varphi}=\frac{(1-K)}{\left(1-A^{2} Q\right)}, \quad K_{\varphi}=w K$

Integrating (38) we get

$\frac{w^{2}}{2}-\left(\frac{1}{\left(1-A^{2} Q\right)}\right)(\log K-K)=$ constant.

Solution (39) is periodic when $K>0$ and $A^{2} Q<1$. In the limit $A^{2} Q \rightarrow 0$, this solution is similar to that obtained by Venkatachalappa et al. [1,2] for rotating compressible or incompressible fluids.

(iii) $\lambda_{1} \rightarrow 0$ or $\lambda_{2} \rightarrow 0$ :

In the limits $\lambda_{1} \rightarrow 0$ or $\lambda_{2} \rightarrow 0$ with $\lambda_{3}$ finite, Eq. (22) reduces to

$T_{\varphi}=\frac{2 \Omega w}{\lambda_{3}\left(1-A^{2} Q\right)}, \quad w_{\varphi}=\frac{(1-K)}{\left(1-A^{2} Q\right)}, \quad K_{\varphi}=w K$.

From (40), we obtain the integral

$K=\exp \left[\frac{2 \Omega T}{\lambda_{3}\left(1-A^{2} Q\right)}\right]$.

The phase curves in the $(w, K)$ plane are similar to those for the limiting case $\lambda_{3} \rightarrow \infty$.

\section{Conclusion}

For linear case the solutions are periodic in $\left(w^{\prime}, K^{\prime}\right)$ plane when either the waves travel in the horizontal direction in the limit $\lambda_{3} \rightarrow \infty$ or in the vertical direction when either $\lambda_{1} \rightarrow 0$ or $\lambda_{2} \rightarrow 0$; the periodic nature of the solution in these limits is not affected by the presence of Earth's rotation provided that $A^{2} Q<1$. This is in contrast to what is observed for the compressible medium. It is found that in the latter case (see Venkatachalappa et al. [1]) the condition for the existence of horizontally propagating periodic waves is $n_{1}<\bar{n}<n_{2}$ (see Eq. (33)). However, in the incompressible limit $\gamma \rightarrow \infty, n_{1}$ and $n_{2}$ tend to zero; therefore, the window of non-periodicity disappears and we have periodic solutions. In $\left(T^{\prime}, w^{\prime}, K^{\prime}\right)$ space, the waves are aperiodic in view of integral (35) which gives linear relation between $T^{\prime}$ and $K^{\prime}$.

When non-linear effects are included, the solutions are periodic in $(w, K)$ plane when either the waves propagate horizontally, or verticall for both rotating and non-rotating systems and is true for conducting fluids provided that $A^{2} Q<1$. All these solutions, however, are non-periodic in the $(T, w)$ plane. We observe from (41) that $K$ varies exponentially with $T$. Thus in general the solutions in the $(T, w, K)$ space are non-periodic but they exhibit periodic nature when they propagate only in the horizontal direction.

\section{Acknowledgement}

This work is dedicated to (Late) Prof. P.L. Sachdev, INSA Senior Scientist, Department of Mathematics, Indian Institute of Science, Bagalore 560 012, India. 


\section{References}

[1] M. Venkatachalappa, N. Rudraiah, P.L. Sachdev, Propagation of quasi-simple waves in a compressible rotating atmosphere, Acta Mech. 88 (1991) 153-166.

[2] M. Venkatachalappa, L.N. Achala, P.L. Sachdev, Exact non-linear travelling waves in rotating systems, Acta Mech. (2000) 1-11.
[3] M. Venkatachalappa, N. Rudraiah, P.L. Sachdev, Exact nonlinear travelling hydromagnetic wave solutions, Acta Mech. 93 (1992) 1-11.

[4] V.S. Seshadri, P.L. Sachdev, Quasi-simple wave solutions for acoustic gravity waves, Phys. Fluids 20 (1977) 888-894. 